\title{
Semiclassical quantization of the diamagnetic hydrogen atom with near action-degenerate periodic-orbit bunches
}

\author{
Jan Gehrke, Jörg Main, and Günter Wunner \\ Institut für Theoretische Physik 1, Universität Stuttgart, 70550 Stuttgart, Germany
}

(Dated: November 8, 2018)

\begin{abstract}
The existence of periodic orbit bunches is proven for the diamagnetic Kepler problem. Members of each bunch are reconnected differently at self-encounters in phase space but have nearly equal classical action and stability parameters. Orbits can be grouped already on the level of the symbolic dynamics by application of appropriate reconnection rules to the symbolic code in the ternary alphabet. The periodic orbit bunches can significantly improve the efficiency of semiclassical quantization methods for classically chaotic systems, which suffer from the exponential proliferation of orbits. For the diamagnetic hydrogen atom the use of one or few representatives of a periodic orbit bunch in Gutzwiller's trace formula allows for the computation of semiclassical spectra with a classical data set reduced by up to a factor of 20 .
\end{abstract}

PACS numbers: 32.60.+i, 03.65.Sq, 05.45.-a

\section{INTRODUCTION}

Semiclassical theories provide the link between quantum spectra and the dynamics of the underlying classical system. For systems with chaotic classical dynamics Gutzwiller's trace formula [1] expresses the density of states in terms of parameters of the isolated unstable periodic orbits. Chaotic systems typically exhibit an exponential proliferation of the number of periodic orbits with growing length. The periodic-orbit sum of Gutzwiller's trace formula usually does not converge, and special techniques as, for example, the cycle expansion [2] or harmonic inversion [3 5] method must be applied for the computation of semiclassical eigenvalues from a finite set of trajectories. Although these techniques can be very powerful for certain systems a remaining conceptually weak point of periodic orbit quantization is still that, due to the exponential proliferation of orbits, the gradual improvement or extension of semiclassical spectra usually requires an exponentially increasing set of periodic orbit data. In this article we propose a way to significantly reduce the number of trajectories which is necessary for periodic orbit quantization by employing properties of the orbits which have recently been investigated in connection with universal features of quantum chaos.

According to the Bohigas-Giannoni-Schmit conjecture [6] a signature of quantum chaos is universal spectral fluctuations on the scale of the mean level spacing. For example, for systems with time reversal symmetry the spacings show a Wigner distribution. On the way towards an understanding or even proof of the conjecture the correlations between orbits which play an important role in the semiclassical approximation to the spectral form factor have been studied 7-13. Strong correlations exist only between orbits which have identical or near identical actions. It has been found that periodic orbits consist of different segments 14. In each segment, an orbit follows closely its neighboring orbit or the time-reverse of this orbit, but the orbits differ in how these segments are connected at self-encounters. As a consequence long periodic orbits of hyperbolic systems do not exist as independent individuals but rather come in closely packed near action-degenerate periodic-orbit bunches. Pairs of orbits with two differently connected loops provide the leading off-diagonal contributions to the form factor [7, 8]. The role of self-encounters and periodic-orbit bunches for universal level correlations in quantum chaos has been investigated in detail in Refs. 9.13.

Here we want to demonstrate that near actiondegenerate periodic-orbit bunches can help to significantly improve the efficiency of semiclassical quantization methods. The idea is not to use all individual periodic orbits up to a given length but only one or very few representatives of a periodic-orbit bunch. Those representatives are appropriately weighted in the periodic orbit sum according to the size of the bunch, which can be determined, for systems with a known symbolic code, solely on the level of the symbolic dynamics without a numerically expensive periodic-orbit search.

Results are presented for the hydrogen atom in a magnetic field, which has a long history as a prototype model of a real physical system with signatures of quantum chaos [15, 16]. At sufficiently high positive energies it is an open system with fully hyperbolic classical dynamics, and a unique symbolic code for the periodic orbits does exist [17-19]. Semiclassical resonances of the diamagnetic hydrogen atom have already been obtained with a modified and extended cycle expansion technique [20.

The article is organized as follows. In Sec. II we discuss the classical dynamics of the diamagnetic hydrogen atom and present an efficient multi-shooting algorithm for the numerical periodic-orbit search. In Sec. III we reveal the existence of periodic-orbit bunches and introduce four reconnection rules which allow for the grouping of orbits on the level of the symbolic dynamics. Semiclassical resonances are computed in Sec. IV with the harmonic inversion method, and it is illustrated how using periodicorbit bunches significantly improves the efficiency of the method. Concluding remarks are given in Sec. $\mathrm{V}$. 


\section{CLASSICAL DYNAMICS AND PERIODIC-ORBIT SEARCH}

In this section we recapitulate the basic equations for the classical dynamics of the diamagnetic hydrogen atom and present a very efficient multi-shooting algorithm for the periodic orbit search.

In atomic units the Hamiltonian of the diamagnetic hydrogen atom reads

$$
H=\frac{1}{2} \boldsymbol{p}^{2}-\frac{1}{r}+\frac{1}{2} \gamma L_{z}+\frac{1}{8} \gamma^{2}\left(x^{2}+y^{2}\right)=E,
$$

where $\gamma=B /\left(2.35 \times 10^{5} \mathrm{~T}\right)$ with $B$ the magnetic field strength. We only consider states with magnetic quantum number $m=L_{z}=0$. The classical dynamics does not depend separately on the energy $E$ and the magnetic field strength $\gamma$ but only on the scaled energy $\tilde{E}=E \gamma^{-2 / 3}$. Using a regularization of the Coulomb singularity in scaled semiparabolical coordinates [15, 16,

$$
\mu=\gamma^{1 / 3} \sqrt{r+z} \text { and } \nu=\gamma^{1 / 3} \sqrt{r-z},
$$

the classical equations of motion in the transformed time $\tau$, with $d \tau=2 r d t$, are derived from the scaled and regularized Hamiltonian

$$
h=\frac{1}{2}\left(p_{\mu}^{2}+p_{\nu}^{2}\right)+V(\mu, \nu)=2,
$$

with the potential

$$
V(\mu, \nu)=-\tilde{E}\left(\mu^{2}+\nu^{2}\right)+\frac{1}{8}\left(\mu^{4} \nu^{2}+\mu^{2} \nu^{4}\right) .
$$

The numerical computation of periodic orbits is a prerequisite for the semiclassical quantization of chaotic systems. In chaotic billiards with an existing symbolic dynamics such as the three-disk scatterer or the hyperbola billiard, a periodic orbit with given symbolic code can be computed efficiently by moving the reflection points until the orbit length becomes a minimum. For systems with smooth potentials the periodic orbit search is more difficult. Using a simple shooting algorithm trajectories with varying starting points are integrated numerically until the initial and final point match. When applying this method for a systematic periodic orbit search some orbits are typically found many times while others may be overlooked. In particular, long and very unstable orbits are hard to find.

For the computation of the periodic orbits of the diamagnetic hydrogen atom we employ a multi-shooting algorithm which is adapted to the symbolic dynamics of the system. With this algorithm we can find selectively each orbit corresponding to a given symbolic code, even when the orbit is very long and unstable.

If one allows the semiparabolic coordinates $(\mu, \nu)$ to be positive or negative, the potential (4) has a $C_{4 v}$ symmetry. Periodic orbits can be described by a ternary symbolic code [17 in a similar way as for the hyperbola or

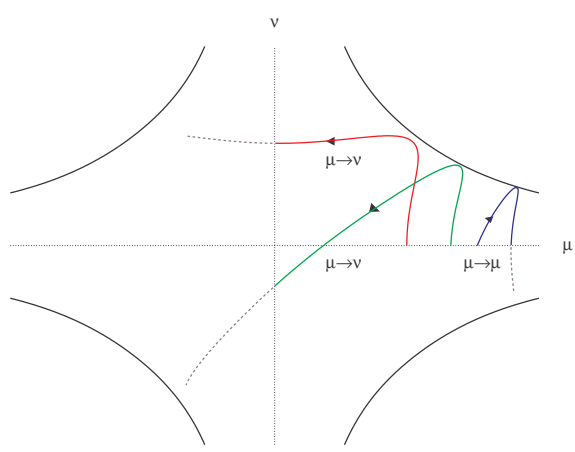

FIG. 1. (Color online) In the multi-shooting algorithm each orbit is split into short segments starting and ending on one of the axes. The concatenation of various types of segments is guided by the symbolic code of the orbit.

four-disk billiard with the same symmetry. At high energies $\tilde{E}>\tilde{E}_{c}=0.329$ there is a one-to-one correspondence between orbits and the symbolic code, whereas below the critical energy orbits undergo bifurcations and the symbolic dynamics is pruned [18, 19].

The multi-shooting algorithm for the periodic orbit search basically works as follows. Each periodic orbit is split into segments, with the number of segments equal to the string length $L$ of the symbolic code. The segments start on either the $\mu$ or the $\nu$ axis and end on one of the axes as illustrated in Fig. 1. When we start all segments with initial guesses chosen in accordance with the symbolic code the orbit is discontinuous between the end point of one and the starting point of the next segment. Now the initial conditions of all segments are iteratively changed to remove the discontinuities. This problem can be formulated as a $2 L$-dimensional root search. (Note that two parameters can be changed for each segment to vary the initial conditions on one of the axes.)

Because the segments are short the multi-shooting algorithm usually converges rapidly and uniquely to the periodic orbit selected by the symbolic code, provided the orbit is not pruned or very close to bifurcation. The method works very well even for very long and unstable orbits.

An example of a periodic orbit at scaled energy $\tilde{E}=0.5$ is presented in Fig. 2, For the symbolic code of a periodic orbit we adopt the ternary alphabet of Ref. [17. with a slightly modified way of the symmetry reduction. In the full coordinate space [see Fig. 2(a)] where the semiparabolic coordinates are extended to negative values each orbit is labeled by a sequence of numbers 1 to 4 for the reflections at the potential barrier in one of the quadrants. The $C_{4 v}$ symmetry of the potential allows for symmetry reduction of the symbolic code. Symbols $\mu$, $\nu$, and 0 are used for the orbit crossing the $\mu$ - or $\nu$-axis in a clockwise or anticlockwise turn, or moving to the opposite potential barrier with a crossing of both axis. At this level of symmetry reduction orbits are located in 

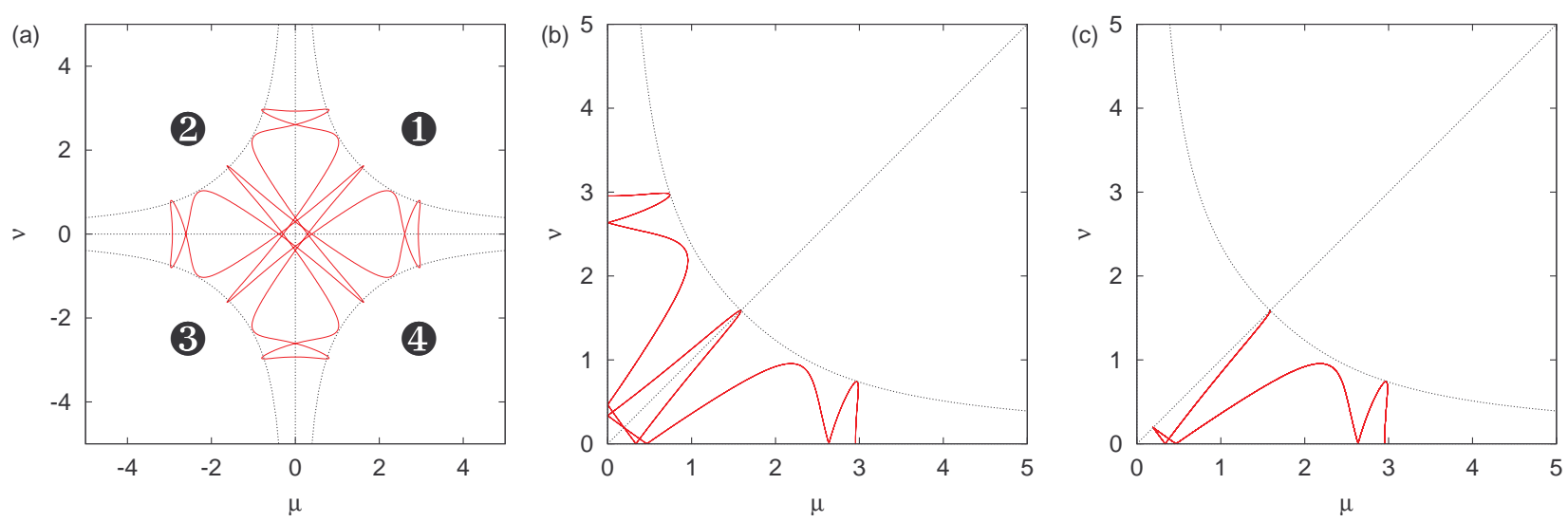

FIG. 2. (Color online) Example of a periodic orbit displayed in (a) the full coordinate plane, (b) the 1st quadrant, and (c) the fundamental domain.

the first quadrant [see Fig. 2(b)]. Finally, an axis symbol $\mu$ or $\nu$ is replaced with a + or - sign when it follows a different or the same axis symbol (ignoring any intermediate 0 symbols), respectively. This alphabet describes the symmetry reduced orbit in the fundamental domain [see Fig. 2(c)]. The various levels of symmetry reduction are helpful to illustrate the periodic orbit bunches and reconnection rules in Sec. III,

\section{PERIODIC-ORBIT BUNCHES}

In this section we first provide an example of a group of near action-degenerate orbits of the diamagnetic hydrogen atom. We then present four reconnection rules which are based on the symbolic dynamics of the periodic orbits and can be used for grouping the trajectories in periodic-orbit bunches. The properties of the periodicorbit bunches are discussed.

\section{A. Example of a near action-degenerate periodic-orbit bunch}

We start our discussion of periodic-orbit bunches of the diamagnetic hydrogen atom by way of example of a group of 16 orbits with cycle length $L=10$ at scaled energy $\tilde{E}=0.5$. The orbits are presented in Fig. 3 in the fundamental domain of the coordinate space. In the overview the similarities between the orbits are quite obvious. All orbits appear to run nearly parallel and thus to be located in the same area of the phase space. For a more detailed discussion the 16 orbits of the periodic-orbit bunch are drawn separately in Fig. 4 in semiparabolic $(\mu, \nu)$ coordinates without symmetry reduction along the $\mu=\nu$ symmetry line. Each orbit is labeled by the symbolic code using the ternary alphabet introduced in Sec. II To make the graphs concise only one fundamental period is shown in Fig. 4, i.e., some orbits are periodic only in the fully symmetry reduced fundamental domain. At first glance

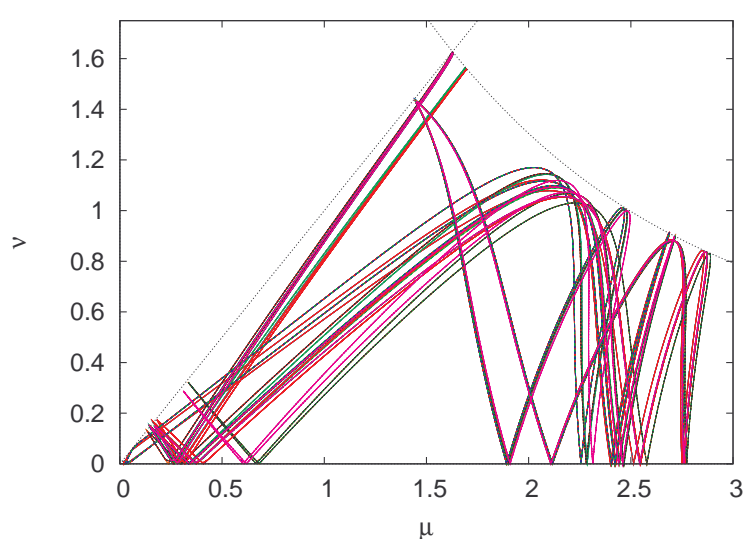

FIG. 3. (Color online) Example of a periodic-orbit bunch consisting of 16 near action-degenerate orbits drawn in the fundamental domain of the $(\mu, \nu)$ coordinate space for scaled energy $\tilde{E}=0.5$. The similarities between orbits are obvious in the overview.

the orbits in Fig. 4 do not appear to be very similar, however, the similarities are obvious in Fig. 3 after complete symmetry reduction in the fundamental domain of the coordinate space. A more detailed inspection of Fig. 4 allows us to reveal both the similarities and differences of the members of the periodic-orbit bunch and to illustrate the reconnection rules.

The basic mechanism for the formation of near actiondegenerate periodic-orbit bunches is that each periodic orbit can be split into segments where the starting and end points of segments can be connected in various ways using simple combinatorial rules. Two types of segments exist where the trajectory is located either close to or far away from the $\mu=\nu$ symmetry line. They can be described most efficiently by the symbolic code of the orbits. A sequence of one or more zero symbols followed by one plus or minus symbol characterizes a segment located near the $\mu=\nu$ symmetry line, a sequence of plus and minus symbols (without any zero) characterizes a segment 
4

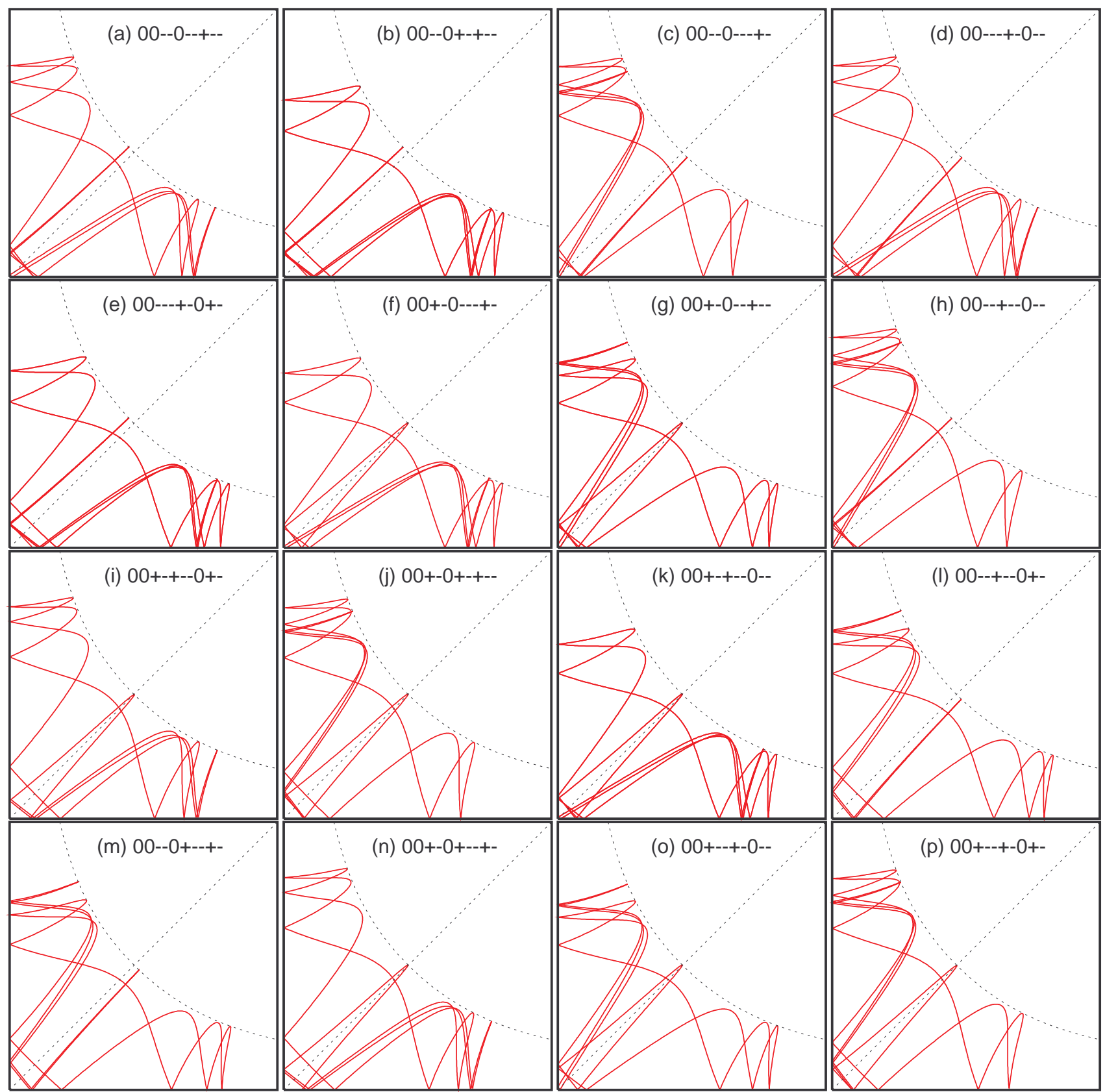

FIG. 4. (Color online) Example of a near action-degenerate periodic-orbit bunch at scaled energy $\tilde{E}=0.5$ consisting of 16 orbits with cycle length $L=10$. The trajectories are drawn in semiparabolic $(\mu, \nu)$ coordinates and are labeled by the symbolic code. See text for discussion.

away from the symmetry line. All orbits in Fig. 4 thus consist of four segments, erg., 00-|-|0-|-+-- in Fig. 4(a). The orbits in Fig. 4(b)-(p) are obtained from the orbit in Fig. 4(a) by reconnecting the four segments in variou ways. For example, in the orbit $00-|-+--| 0-\mid-$ in Fig. $4(\mathrm{~h})$ the two segments - and -+-- are interchanged, and in the orbit $00-|-| 0-\mid--+-$ in Fig. 4 (c) the segment -+-- is traversed backward. Furthermore, the plus or minus symbol following a sequence of zero symbols can be interchanged [see orbits $00-|-| 0+|-+--, 00+|-|0-|-+--$, and $00+|-| 0+\mid-+--$ in Fig. $4(b),(g)$, and $(\mathrm{j})]$ which is related to the mirroring of segments at the $\mu=\nu$ symmetry line. The remaining orbits in Fig. 4 are obtained by combined application of several reconnection rules, e.g., in Fig. 4 $(\mathrm{k})$ the segments - and -+-- are interchanged and the first segment $00-$ is replaced with $00+$. 


\section{B. Reconnection rules}

We denote a sequence of one or more zero symbols followed by one plus or minus symbol as (0)-stretch, and an arbitrary sequence of plus and minus symbols without any zero symbol as (+-)-stretch. The grouping of trajectories of the diamagnetic hydrogen atom in periodic-orbit bunches can now be described by the following four reconnection rules.

Rule $R$-1: The last symbol of a (0)-stretch, which is a plus or minus symbol, can be replaced with the other one.

Rule R-2: The order of symbols in a (+-)-stretch can be reversed.

Rule $R$-3: In a symbolic code with two or more (0)stretches different (0)-stretches can be interchanged.

Rule $R$-4: In a symbolic code with two or more (+-)stretches different (+-)-stretches can be interchanged.

To illustrate the reconnection rules we discuss once more the periodic orbits shown in Fig. 4. The first orbit 00--0--+-- in Fig. 4 (a) can be decomposed into the (0)stretch 00-, the (+-)-stretch -, the (0)-stretch 0-, and the (+-)-stretch -+--. The remaining 15 orbits in Fig. 4 can be obtained from that orbit by application of one or more of the reconnection rules. For example, the orbit in Fig. 4(b) is obtained by applying rule R-1 to the second (0)-stretch 0-, the orbit in Fig. 4(c) is obtained by applying rule R-2 to the (+-)-stretch-+--, and the orbit in Fig. $4(\mathrm{~d})$ is obtained by applying the rules R-2 and R-3, i.e., the (+-)-stretch -+-- is reversed and then the two (+-)-stretches --+- and - are interchanged. Applications of the reconnection rules mean that parts of the orbit drawn in semiparabolic $(\mu, \nu)$ coordinates are approximately mirrored at the $\mu=\nu$ symmetry line, parts of the orbit are traversed backward, or parts of the orbit are visited in a different order. However, in the fundamental domain of the $(\mu, \nu)$ coordinate space the global shape of orbits belonging to the same periodic-orbit bunch is very similar, as can be clearly seen in Fig. 3 .

The reconnection rules are schematically illustrated in Fig. 5. A periodic-orbit bunch is characterized by a set of $k(+-)$-stretches, which is a sequence of plus and minus symbols (an empty set is allowed), and (for $k \geq 2$ ) the same number of (0)-stretches. The (0)-stretches are located in the vicinity of the $\mu=\nu$ symmetry line and are indicated by red solid lines in Fig. 5. The (+-)-stretches are located in regions with $\mu \neq \nu$ away from the symmetry line and are indicated by green dashed lines. Each (0)-stretch is followed by a (+-)-stretch and vice versa, the connections are indicated by the thin black lines in Fig. 5. The individual members of the periodic-orbit bunch differ in the way how the stretches are connected: (+-)-stretches can be mirrored at the $\mu=\nu$ symmetry line (rule R-1), traversed backward (rule R-2), and the orders of the (0)- or (+-)-stretches can be interchanged (rules R-3 and R-4).

How many orbits belong to a certain periodic-orbit bunch? Although we cannot give a precise answer it is

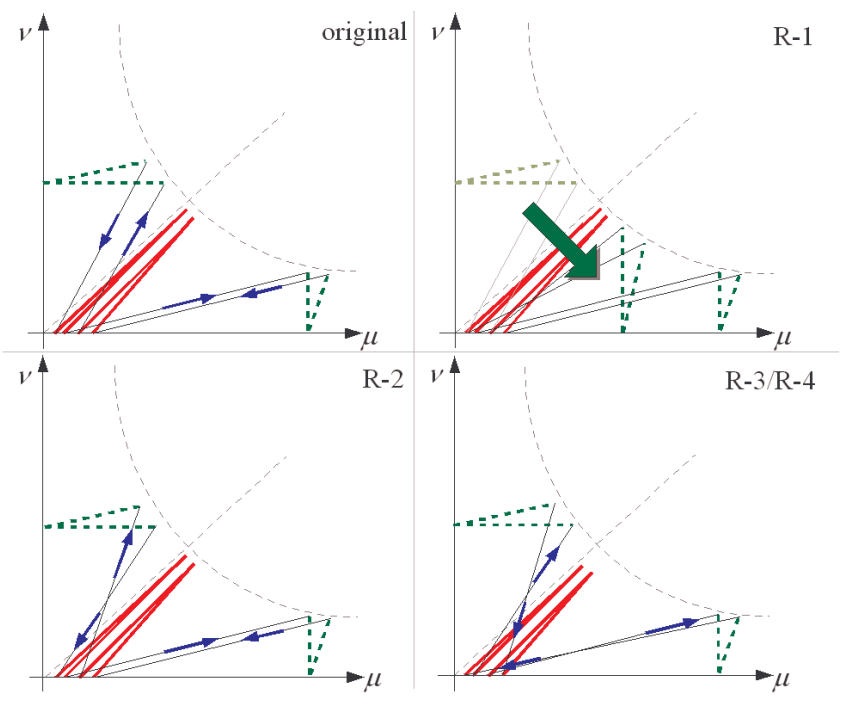

FIG. 5. (Color online) Sketch of the four reconnection rules. In the $(\mu, \nu)$ coordinate space (0)-stretches indicated by red solid lines are located in the vicinity of the $\mu=\nu$ symmetry line, and (+-)-stretches indicated by green dashed lines are located in regions with $\mu \neq \nu$ away from the symmetry line. The connections between (0)- and (+-)-stretches are drawn with thin black lines. (+-)-stretches can be mirrored at the $\mu=\nu$ symmetry line (rule R-1), traversed backward (rule $\mathrm{R}-2$ ), and the orders of the (0)- or (+-)-stretches can be interchanged (rules R-3 and R-4).

easy to estimate an upper bound of that number from the combinatorial reconnection rules. Let again $k$ be the number of (+-)- and (0)-stretches in a symbolic code. According to rule R-1 the last symbol of a (0)-stretch, which is + or -, can be replaced with the other one, resulting in $2^{k}$ possibilities. According to rule R-2 the order of symbols in a (+-)-stretch can be reversed. Each $(+-)$-stretch which is not symmetric under that transform provides a factor of two, resulting in at most $2^{k}$ possibilities. The interchange of (0)-stretches and (+-)stretches according to rules R-3 and R-4 provide at most (substrings may be identical) $k$ ! and $(k-1)$ ! possibilities, respectively, where, in the second factorial, it has been taken into account that a cyclic permutation of all (0)and (+-)-stretches does not change the periodic orbit. Altogether we obtain a maximum number of orbits

$$
N_{k}^{\max }=k !(k-1) ! 2^{2 k}
$$

building one periodic-orbit bunch. The 16 orbits shown in Fig. 4 have $k=2(0)$-stretches. The number of orbits is less than the maximum $N_{2}^{\max }=32$ because the (+-)stretch consisting of a single minus symbol coincides with its reverse. The maximum number $N_{k}^{\max }$ increases very rapidly with $k$, e.g., for $k=4$ bunches with up to $N_{4}^{\max }=$ 36864 orbits exist. However, for such a huge bunch the four (0)-stretches must have different lengths and the four (+-)-stretches including their time reversals must be different, which is found to be possible only for very long orbits with code lengths $L \geq 26$. 


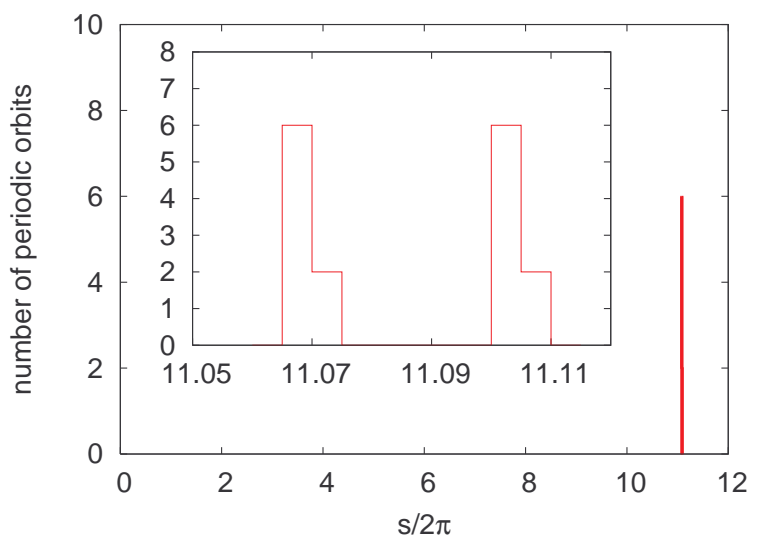

FIG. 6. (Color online) Distribution histogram of the classical actions for the 16 trajectories of the near action-degenerate periodic-orbit bunch shown in Figs. 3 and 4 . The small differences between the actions of individual orbits are only visible in the enlarged inset.

\section{Properties of periodic-orbit bunches}

The most important feature of the periodic-orbit bunches is that the individual trajectories have similar shape in coordinate space (see Fig. 3), and thus all orbits have nearly the same action and stability properties. For the 16 orbits shown in Figs. 3 and 4 the near degeneracy of the actions is illustrated in Fig. 6. In low resolution the distribution of the actions exhibits a single peak at $s / 2 \pi \approx 11.1$. Only in high resolution (see the inset in Fig. 6) the distribution of the actions in a small but nonzero range $\Delta s$ can be observed.

For a given periodic-orbit bunch the action range $\Delta s$ basically depends on the number of zero symbols in the shortest (0)-stretch. We illustrate this for the orbit pairs

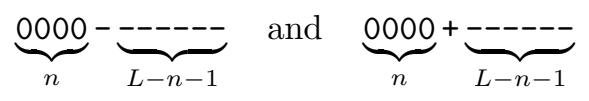

which consist of a (0)-stretch with $n$ zero symbols followed by a plus or minus symbol and a (+-)-stretch with $L-n-1$ minus symbols. The total length of the symbolic code is $L$. For fixed $L$ and $n$ the two orbits belong to the same periodic-orbit bunch (see rule R-1). The action difference $\Delta s$ between the two orbits for various values of $L$ and $n$ is shown in Fig. 7. Evidently, $\Delta s$ decreases exponentially with the number $n$ of consecutive zero symbols but only weakly depends on the total code length $L$. This result does not depend on the special choice of the $(+-)$-stretch as a sequence of minus symbols, i.e., similar results have been obtained for other types of (+-)stretches. A more detailed investigation shows that the similarities of orbits in a periodic-orbit bunch become more and more pronounced with increasing length of the shortest (0)-stretch. The reason is the decrease of the angle or distance of the orbit at self-encounters with increasing length of the (0)-stretches [21].

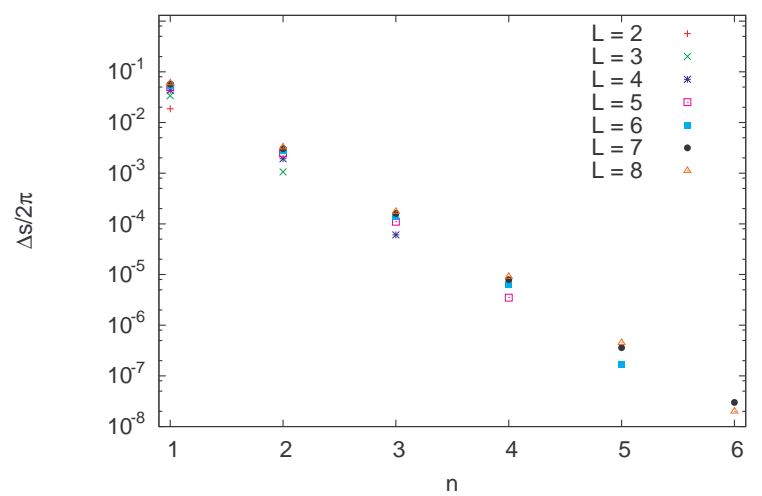

FIG. 7. (Color online) Action difference $\Delta s$ between two orbits consisting of a (0)-stretch with $n$ zero symbols and a (+-)-stretch with $L-n-1$ minus symbols. Evidently, $\Delta s$ decreases exponentially with the length of the (0)-stretch.

\section{SEMICLASSICAL QUANTIZATION WITH PERIODIC-ORBIT BUNCHES}

As already mentioned in Sec. [1 self-encounters and periodic-orbit bunches play an important role for the understanding of the level statistics of quantum systems with an underlying chaotic classical dynamics. We will now demonstrate the relevance of periodic-orbit bunches for the semiclassical quantization of the diamagnetic hydrogen atom.

The energy eigenvalues of integrable classical systems can be obtained by semiclassical torus quantization. In chaotic systems torus structures in phase space are absent. It has been shown by Gutzwiller [1] that the unstable periodic orbits are the skeleton for the semiclassical quantization of chaotic systems. However, the problems with Gutzwiller's trace formula are twofold: First, the periodic orbit sum is not absolutely convergent and special techniques such as cycle expansion [2] or harmonic inversion 3 [5] methods are necessary to obtain converged results. Second, in chaotic systems the number of periodic orbits grows exponentially with the orbital lengths. It is very unsatisfactory that an exponentially increasing set of classical data information is necessary to resolve a few more semiclassical eigenvalues. However, as already mentioned, the required classical data can be significantly reduced when using the periodic-orbit bunches instead of the individual periodic orbits for the semiclassical quantization.

\section{A. Harmonic inversion method}

We employ the harmonic inversion method for periodic orbit quantization [3 [5, 22, and, for the convenience of the reader, here briefly recapitulate its basic ingredients. According to [1] the semiclassical response function of 
chaotic systems is given by

$$
g_{\mathrm{sc}}(E)=g_{\mathrm{sc}}^{0}(E)+\sum_{\mathrm{po}} \mathcal{A}_{\mathrm{po}}(E) e^{i S_{\mathrm{po}}(E)}
$$

where $g_{\mathrm{sc}}^{0}$ is a smooth function of the energy, $S_{\mathrm{po}}$ is the classical action of a periodic orbit, and $\mathcal{A}_{\mathrm{po}}$ is the amplitude of that orbit including phase information. The semiclassical energies or resonances are the poles of the response function $g_{\mathrm{sc}}(E)$ in Eq. (6).

The hydrogen atom in a magnetic field possesses a scaling property, as already introduced in Sec. III i.e., the classical dynamics does not depend separately on the energy $E$ and the magnetic field strength $\gamma$ but only on the scaled energy $\tilde{E}=E \gamma^{-2 / 3}$. Keeping the scaled energy constant the response function can be written as a function of $w=\gamma^{-1 / 3}$, viz.

$$
g_{\mathrm{sc}}(w)=g_{\mathrm{sc}}^{0}(w)+\sum_{\mathrm{po}} \mathcal{A}_{\mathrm{po}} e^{i w s_{\mathrm{po}}},
$$

where $s_{\text {po }}=\gamma^{1 / 3} S_{\text {po }}$ is the scaled action of a periodic orbit and the amplitude

$$
\mathcal{A}_{\mathrm{po}}=\frac{s_{\mathrm{ppo}}}{\sqrt{\left|2-\lambda_{\mathrm{ppo}}^{r}-\lambda_{\mathrm{ppo}}^{-r}\right|}} e^{-i r \mu_{\mathrm{ppo}} \pi / 2}
$$

depends on the scaled action $s_{\text {ppo }}$ of the primitive periodic orbit (ppo) where the orbit is traversed only once, the repetition number $r$ of that orbit, the leading eigenvalue $\lambda_{\text {ppo }}$ of the monodromy matrix, and the Maslov index $\mu_{\mathrm{ppo}}$. The Fourier transform of the scaled response function in Eq. (7) yields the semiclassical signal

$$
C_{\mathrm{sc}}(s)=\frac{1}{2 \pi} \int_{-\infty}^{\infty} g_{\mathrm{sc}}(w) e^{-i s w} d w=\sum_{\mathrm{po}} \mathcal{A}_{\mathrm{po}} \delta\left(s-s_{\mathrm{po}}\right)
$$

which possesses $\delta$-peaks with weight factors $\mathcal{A}_{\text {po }}$ at the actions $s=s_{\text {po }}$ of the classical periodic orbits.

The periodic orbit quantization is achieved by adjusting the semiclassical signal $(9)$ to its quantum analogue. The quantum resonances are the eigenvalues $w=\gamma^{-1 / 3}$ of the scaled Schrödinger equation at constant scaled energy

$$
\begin{aligned}
& {\left[-2 \tilde{E}\left(\mu^{2}+\nu^{2}\right)+\frac{1}{4}\left(\mu^{4} \nu^{2}+\mu^{2} \nu^{4}\right)-4\right] \psi} \\
& =w^{-2}\left(\Delta_{\mu}+\Delta_{\nu}\right) \psi \text { with } \Delta_{\rho}=\frac{1}{\rho} \frac{\partial}{\partial \rho}\left(\rho \frac{\partial}{\partial \rho}\right) .
\end{aligned}
$$

The Fourier transform of the quantum mechanical Green's function,

$$
g_{\mathrm{qm}}(w)=\sum_{k} \frac{d_{k}}{w-w_{k}}
$$

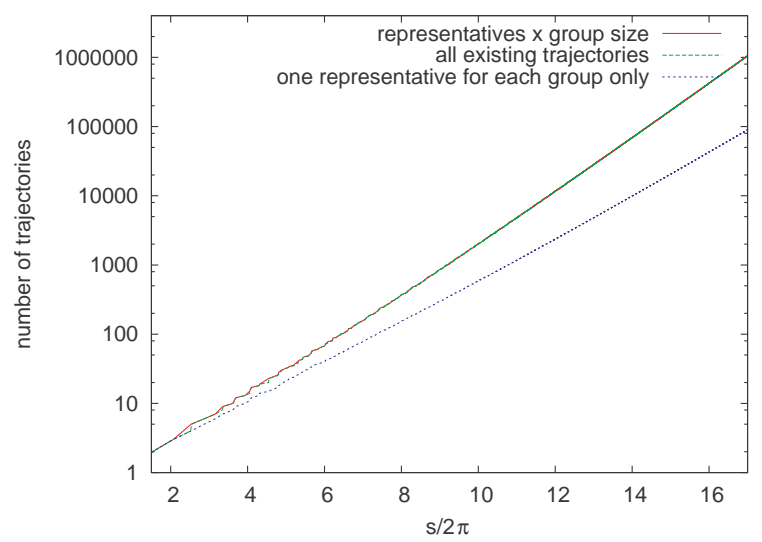

FIG. 8. (Color online) Number of periodic orbits with classical action $s_{\text {po }}<s$ for all individual orbits (dashed green line), only one representative of a periodic-orbit bunch (dotted blue line), and one representative of a periodic-orbit bunch weighted with the size of the bunch (solid red line, nearly indistinguishable from the dashed green line). All curves show an exponential increase, however, the number of periodicorbit bunches grows with a significantly lower rate than the number of all orbits.

with resonances in the lower complex half plane, i.e., $\operatorname{Im} w_{k}<0$ and residuals $d_{k}=1$ for non-degenerate states yields the quantum signal

$$
C_{\mathrm{qm}}(s)=\frac{1}{2 \pi} \int_{-\infty}^{\infty} g_{\mathrm{qm}}(w) e^{-i s w} d w=-i \sum_{k} d_{k} e^{-i w_{k} s} .
$$

The semiclassical signal (9) obtained from the classical periodic orbit data is now adapted to the quantum signal (12), with the $d_{k}$ and $w_{k}$ being free adjustable complex parameters by means of nonlinear signal processing methods. Technical details can be found in Refs. [22, 23].

The periodic orbit signal $C_{\mathrm{sc}}(s)$ must be known in the interval $0<s<s_{\max }$. The required signal length $s_{\max }$ is proportional to the density of eigenstates $w_{k}$, which usually increases for higher excitations, i.e., an increasing signal length is required to resolve the denser parts of the quantum spectrum.

\section{B. Reduced data set}

Since in classically chaotic systems the number of periodic orbits grows exponentially with the orbital period or action, a linear increase of the length $s_{\max }$ of the periodic orbit signal (9) requires an exponential increase of the classical input data and the numerical effort for the periodic-orbit search. For the hydrogen atom in a magnetic field at scaled energy $\tilde{E}=0.5$ the exponential increase of the number of trajectories with growing action is illustrated by the dashed green line in Fig. 8 For the construction of the periodic-orbit signal (9) with signal length $s_{\max } / 2 \pi=17$ more than one million periodic 
orbits must be computed.

To reduce the required amount of periodic-orbit data we take advantage of properties of the periodic-orbit bunches. Since all orbits of a bunch are near actiondegenerate (cf. Fig. 6) and also have very similar stability properties and identical Maslov indices the parameters $\mathcal{A}_{\text {po }}$ and $s_{\text {po }}$ in Eq. (9) are approximately the same for all members of a periodic-orbit bunch. (Additional weight factors which arise from symmetry decomposition will be discussed in Sec. IV C) The idea now is not to compute all members but only one representative (or very few representatives) of a periodic-orbit bunch, and to use the actions and appropriately weighted amplitudes of the representatives for the construction of the periodicorbit signal. The Maslov index which determines the complex phase of the amplitudes in Eq. (8) can be obtained directly and without any numerical periodic orbit search from the symbolic dynamics of orbits and reads $\mu_{\text {po }}=3 L-N_{+}-N_{-}$, where $L$ is the code length and $N_{ \pm}$are the numbers of plus and minus symbols in the code. Note that the Maslov index does not change by application of any of the four reconnection rules.

The construction of the reduced data set is achieved as follows. We need the symbolic dynamics of all orbits and the periodic-orbit parameters of representatives up to a certain action $s_{\max }$. In our computations we use $s_{\max } / 2 \pi=20$. In a first step we calculate the symbolic sequences of orbits. As some orbits with very long symbolic dynamics can contribute to the periodic-orbit signal the computing time is reduced by optimizing the order of generating the symbolic codes in a way that orbits with short action are preferably obtained earlier than orbits with long action. The length limit of every symbolic sequence describing one periodic orbit is achieved if the approximate estimate of the action based upon the symbolic sequence of every orbit exceeds the action limit $s_{\max }$. From the symbolic code of a periodic orbit its action can roughly be estimated by weighting the actions of the fundamental orbits $0,+$, and - with the corresponding number of symbols in the code. We can calculate efficiently up to a maximum code length $L=16$, i.e., the orbit data set is complete up to this length. Longer symbolic sequences are generated by adding minus symbols to shorter symbolic sequences. This does not change the shape of the orbits or the size of the groups in the most cases but adds only new loop parts in outward direction to the periodic orbits. In order to decrease computing time the same method is used to calculate all periodic orbits.

We use the reconnection rules to group the periodic orbits. Arranging the orbits is a much faster procedure than the calculation of the actions because it is based only on integer arithmetic, which is very fast to handle on the computer. As the parameters of the periodic orbits in one group are very similar, we can choose one orbit as representative of the group and calculate all parameters of this trajectory. The results with appropriate weighting are then used for the construction of the periodic-orbit signal.

The number of periodic-orbit bunches with action $s_{\mathrm{po}}<s$ is shown as the dotted blue line in Fig. 8. Similar to the total number of orbits the number of the bunches also grows exponentially with the action, but with a significantly lower rate. For example at $s / 2 \pi=17$ there are only about 80000 bunches, compared to more than one million individual periodic orbits.

\section{Symmetry decomposition}

The diamagnetic hydrogen atom possesses a discrete symmetry, viz. the reflection at the $z=0$ plane, and thus the quantum system has resonances $w_{k}^{ \pm}$in the decomposed subspaces with even and odd $z$ parity. In periodicorbit theory the symmetry decomposition is achieved by multiplying the amplitudes $\mathcal{A}_{\text {po }}$ in Eq. (8) with weight factors $\sigma_{\mathrm{po}}$, which depend on the chosen subspace and symmetry properties of the periodic orbits [24]. The weight factors are

$$
\sigma_{\mathrm{po}}= \begin{cases}1 & \text { for even parity } \\ (-1)^{N_{+}} & \text {for odd parity }\end{cases}
$$

with $N_{+}$the number of plus symbols in the symbolic code. In spectra with even parity all members of a periodic-orbit bunch have identical weight factors $\sigma_{\mathrm{po}}=$ 1 and contribute with approximately the same amplitudes $\mathcal{A}_{\mathrm{po}}$ to the semiclassical signal in Eq. (9), i.e., all orbits of a periodic-orbit bunch interfere constructively and the total weight of the bunch amplitude is approximately the amplitude of a representative multiplied with the number of orbits in the bunch. In odd parity spectra the weight factors $\sigma_{\mathrm{po}}$ of two orbits which are related by a single application of the reconnection rule R-1 have different sign and thus the contributions of the two orbits to the semiclassical signal in Eq. (9) approximately cancel. The total weight of the bunch is the nontrivial sum of the $\sigma_{\text {po }}$ of all individual orbits of the bunch, however, that weight can be obtained solely from the symbolic dynamics of orbits.

\section{Results and discussion}

For the hydrogen atom in a magnetic field exact quantum and semiclassical resonances have already been compared by Tanner et al. 20]. The quantum resonances were obtained by numerical diagonalization of the generalized eigenvalue problem (10) using a complex rotated complete basis set. The semiclassical resonances were computed with a modified and extended cycle expansion technique. As already mentioned at sufficiently high energies $\tilde{E}>\tilde{E}_{c}=0.329$ the symbolic dynamics is complete without any pruning of orbits, however, trajectories going far away from the nucleus in the direction of the magnetic field axis were found to be marginally stable. 

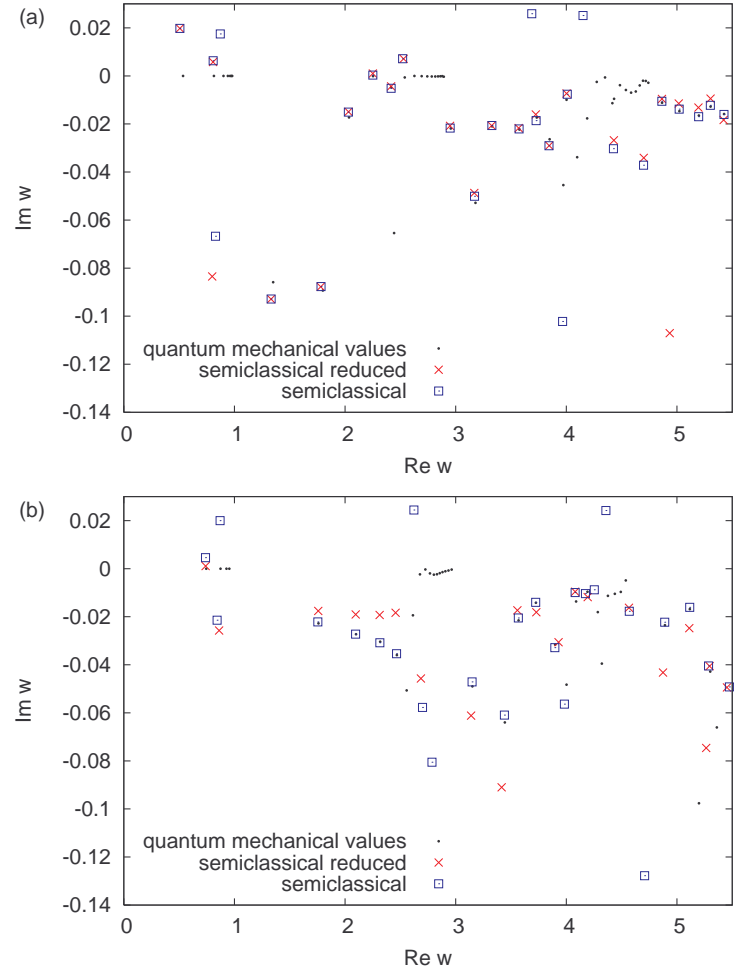

FIG. 9. (Color online) Resonances with (a) even and (b) odd parity of the diamagnetic hydrogen atom at scaled energy $\tilde{E}=0.5$. In most cases especially the real parts of the semiclassical resonances obtained with the reduced data set (red crosses) agree very well with the semiclassical resonances obtained with the complete periodic-orbit set (blue squares). For completeness and comparison the numerically exact quantum resonances are shown as black dots.

Furthermore, resonances accumulate at the thresholds of the Landau channels. For these reasons the periodic orbit quantization of the diamagnetic hydrogen atom is a very nontrivial and challenging task.

Here we apply the harmonic inversion method introduced in Sec. IVA for period-orbit quantization. With a finite length signal this method can resolve sufficiently isolated resonances in the spectra but not the accumulation of resonances at the Landau thresholds. However, we do not focus on the comparison of the quantum and semiclassical resonances but on the results of the two semiclassical quantizations obtained with either the complete periodic-orbit set or with the reduced data set of the periodic-orbit bunches introduced in Sec. IVB.

Resonances with even and odd parity of the diamagnetic hydrogen atom at scaled energy $\tilde{E}=0.5$ are presented in Fig. 9, The semiclassical resonances have been obtained by harmonic inversion of the periodic-orbit sig- nal $C_{\mathrm{sc}}(s)$ with $s_{\max } / 2 \pi=20$ using either the complete periodic-orbit set (blue squares in Fig. 9) or the reduced data set (red crosses). In the reduced data set we have used the two representatives with maximum or minimum action of each periodic-orbit bunch, which can be identified by their symbolic codes. The numerically exact quantum resonances are shown by black dots in Fig. 9 .

As mentioned above the harmonic inversion of a finite length signal cannot reproduce resonances close to the accumulation points at, e.g., $w=1$ and $w=3$. For most of the semiclassical resonances with even parity in Fig. 9. (a) the agreement is excellent for both the real and imaginary parts of the resonances. For the resonances with odd parity in Fig. 9(b) the agreement of the imaginary parts is less perfect, however, the real parts still agree very well. The reason for the larger deviations in spectra with odd parity might be that the possible cancellation of periodic orbit contributions in this subspace is more critical for the accuracy of the periodicorbit signal than the solely constructive superposition of the contributions in the subspace with even parity. The results clearly demonstrate that the use of near actiondegenerate periodic-orbit bunches allows for the reduction of the classical data set by more than an order of magnitude and thus significantly increases the efficiency of periodic-orbit quantization methods.

\section{CONCLUSION}

The existence of periodic-orbit bunches in the classically chaotic diamagnetic hydrogen atom has been revealed. The orbits of a bunch have a similar shape in the fundamental domain of the coordinate space and only differ in the behavior at self-encounters. We have introduced four reconnection rules which allow for the grouping of orbits already on the level of the symbolic dynamics in terms of a ternary alphabet.

The exponential proliferation of periodic orbits of a classically chaotic system implies that semiclassical methods based on Gutzwiller's trace formula typically require an exponentially growing classical data set to resolve more eigenstates. We have shown that the use of one or few representatives of the near action-degenerate orbits can help to significantly improve the efficiency of semiclassical quantization methods.

A peculiarity of the diamagnetic hydrogen atom is that periodic orbits at positive energies are marginally stable. It will thus be very interesting to investigate the existence of near action-degenerate periodic orbits for other chaotic systems such as $N$-disk or $N$-sphere scatterer, and to study the improvement of semiclassical quantization methods by using the periodic-orbit bunches.
[1] M. C. Gutzwiller, Chaos in Classical and Quantum Mechanics (Springer, New York, 1990).
[2] P. Cvitanović and B. Eckhardt, Phys. Rev. Lett. 63, 823 
(1989).

[3] J. Main, V. A. Mandelshtam, and H. S. Taylor, Phys. Rev. Lett. 79, 825 (1997).

[4] J. Main, V. A. Mandelshtam, G. Wunner, and H. S. Taylor, Nonlinearity 11, 1015 (1998).

[5] J. Main, Phys. Rep. 316, 233 (1999).

[6] O. Bohigas, M. J. Giannoni, and C. Schmit, Phys. Rev. Lett. 52, 1 (1984).

[7] M. Sieber and K. Richter, Physica Scripta T90, 128 (2001).

[8] M. Sieber, J. Phys. A 35, L613 (2002).

[9] S. Müller, S. Heusler, P. Braun, F. Haake, and A. Altland, Phys. Rev. Lett. 93, 014103 (2004).

[10] S. Heusler, S. Müller, P. Braun, and F. Haake, J. Phys. A 37, L31 (2004).

[11] S. Müller, S. Heusler, P. Braun, F. Haake, and A. Altland, Phys. Rev. E 72, 046207 (2005).

[12] S. Heusler, S. Müller, A. Altland, P. Braun, and F. Haake, Phys. Rev. Lett. 98, 044103 (2007).

[13] S. Müller, S. Heusler, A. Altland, P. Braun, and F. Haake, New J. Phys. 11, 103025 (2009).

[14] A. Altland, P. Braun, F. Haake, S. Heusler, G. Knieper, and S. Müller, in Path Integrals - New Trends and Perspectives: Proc. 9th Int. Conf. (Dresden), edited by W. Janke and A. Pelster (World Scientific, Singapore, 2008) p. 40.

[15] H. Hasegawa, M. Robnik, and G. Wunner, Prog. Theor. Phys. Suppl. 98, 198 (1989).

[16] H. Friedrich and D. Wintgen, Phys. Rep. 183, 37 (1989).

[17] B. Eckhardt and D. Wintgen, J. Phys. B 23, 355 (1990).

[18] K. T. Hansen, Phys. Rev. E 51, 1838 (1995).

[19] S. Schneider, diploma thesis, Ruhr-Universität Bochum, 1997 (unpublished).

[20] G. Tanner, K. T. Hansen, and J. Main, Nonlinearity 9, 1641 (1996).

[21] J. Gehrke, diploma thesis, Universität Stuttgart, 2008 (unpublished).

[22] J. Main, P. A. Dando, Dž. Belkić, and H. S. Taylor, J. Phys. A 33, 1247 (2000).

[23] Dž. Belkić, P. A. Dando, J. Main, and H. S. Taylor, J. Chem. Phys. 113, 6542 (2000).

[24] P. Cvitanović and B. Eckhardt, Nonlinearity 6, 277 (1993). 